\title{
34th ANNUAL SASKATCHEWAN CHRISTMAS BIRD COUNT — 1975
}

Compiled by MARY I. HOUSTON, 863 University Drive, Saskatoon, Sask.

"Bird Count day always seems to come and go all too quickly. Again we must say we really enjoyed this year's count. The weather was most cooperative; most ideal conditions. I only wish there were more daylight hours; the day just goes too fast. One of the highlights of this count of course, is our regular noon meal. We head to the nearest Aspen bluff, get a fire going, boil us a tea pail and skewer up some farmer sausage and onions, which is later put between brown bread and cheese. A few cookies and home made chocolates top it off. (A quick but tasty meal in the bush).'

The above quotation from Lloyd Sperling of Dalmeny suggests the true spirit of Christmas Bird Counting. No other group activity has added so much to our knowledge of Saskatchewan bird distribution, as it is conducted at a time of year when few observers otherwise would make comprehensive observations.

Since the weather was generally mild over the Count period this year, counters were out in a record 52 areas (46 localities in 1972 was the next highest number). A total of 68 species was seen on Count days with one additional during the Count period.

Three Glaucous Gulls observed at Gardiner Dam by Wayne and Don Renaud and Bob Godwin added one more species to bring the 34-year total to 123 species with 6 additional species seen in Count period but not on Count day. Another unusual sighting during the Count period was of 1 Gray-crowned Rosy Finch seen at Spring Valley on Dec. 30. This species had only been reported on Christmas Bird Counts twice previously: Charles
F. Holmes had reported 25 at Dollard in 1945 and 3 (2 Gray-crowned and 1 Hepburn's subspecies) in 1946.

Gray Partridge and Sharp-tailed Grouse reports and numbers were again low. Snowy Owls were not generally more numerous, but seemed concentrated in large numbers at Moose Jaw (9), Regina (15) and Tuberose (10).

Saskatoon had a heavy influx of Bohemian Waxwings, reporting 12,442 on Count day, while Regina had 14 species of waterfowl.

ASQuith. Dec. 23; by skis around 80-acre field; 9 species, 59 individuals. - Don, Lynne and Muriel Carlson, Gary Entwistle.

BANGOR. Dec. $31 ; 14$ miles by car and at feeder; mild, calm day; 7 species, 58 individuals. - Mrs. A. Thompson.

BIGgAR. Dec. 24; 131 miles by car in 6 hours, $9-1 / 2$ miles on foot in 5-1/2 hours; temp. $-12^{\circ}$ to $-3^{\circ} \mathrm{C}$, wind SW $0-5 \mathrm{mph}$, clear; $10 \mathrm{~cm}$ snow; 14 species, 1,554 individuals. (Add: Blue Jay, 1, Jan. 3; Snow Bunting, 8, Jan. 1 and 2, and 50, Jan. 4). Ron Chulach, Don Renaud, Wayne Renaud, Guy Wapple (compiler), Rodney Wapple.

BIG GULLY CREEK (15 miles NE of Maidstone). Dec. $19 ; 61$ miles by car in 3 hours, 11 miles on foot in 8 hours; temp. $-20^{\circ}$ to $-5^{\circ} \mathrm{C}$, clear in AM becoming cloudy in PM, wind SE 15 $\mathrm{mph} ; 25 \mathrm{~cm}$ snow; 22 species, 740 individuals. - Wayne Harris, Sheila Lamont.

BORDEN. Dec. $28 ; 43$ miles by car in $2-1 / 2$ hours, 1 mile on foot in $1 / 2$ hour; temp. $-3^{\circ} \mathrm{C}$, wind SW $12 \mathrm{mph}$, partly cloudy; $5 \mathrm{~cm}$ snow; 8 species, 600 individuals. - John, Mary and Stan Shadick.

BROADVIEW. Dec. 23;91 miles by car and 3 miles on foot in 6 hours; temp. $-12^{\circ} \mathrm{C}$, wind light, sunny; 17 species, 529 individuals. (Add: Ruffed Grouse, 4, Dec. 29; Gray Partridge, 9, Dec. 29; Horned Lark, 20, Dec. 25; Blue Jay, 1, Dec. 24). - David Chaskavich and Don Weidl. 
CHELAN. Dec. 26; 1 mile along creek on foot, and about yard; temp. $-3^{\circ} \mathrm{C}$, no wind; $5 \mathrm{~cm}$ snow; 6 species, 26 individuals. - Joy Aspen, Jean Harris.

COLD RIVER. Dec. $20 ; 15$ miles by car and 2 miles on foot along river; clear, calm, $+5^{\circ} \mathrm{C} ; 20 \mathrm{~cm}$ snow; 11 species, $243 \mathrm{in}$ dividuals. - William Haras.

DALMENY. Dec. 26; 24 miles by car in 1/2 hour, 42 miles on foot in 32 hours (4 parties); temp. $-3^{\circ}$ to $0^{\circ} \mathrm{C}$, sunny, light $\mathrm{SE}$ wind; 13 species, 1,192 individuals. - Hon Dong, Curtís and Leslie Johanson, Brian, Gilbert, Lorne and Mara Sperling, Lloyd Sperling (compiler).

DILKE. Dec. $30 ; 33$ miles by car and 2-1/2 miles on foot in $3.1 / 2$ hours and around farmyard; temp. $-5^{\circ} \mathrm{C}$, wind $\mathrm{E} 20-30 \mathrm{mph}$, overcast then snow; 9 species, 263 individuals. (Add: Sharp-tailed Grouse, 6, Dec. 21, and 15, Jan. 4; Snow Bunting, 1,000, Dec. 25, and 1, Dec. 27, Jan. 2). J. B., Margaret, Mr. and Mrs. S. R. Belcher.

DUPEROW-RUTHILDA. Dec. 28; 82 miles by car in $5.1 / 2$ hours, 6 miles on foot in 3 hours; temp. $-8^{\circ}$ to $-3^{\circ} \mathrm{C}$, wind $\mathrm{NW} 0-10 \mathrm{mph}$, clear in AM, overcast in PM; $12 \mathrm{~cm}$ snow; 16 species, 2,089 individuals. (Add: Golden Eagle, 1, Jan. 4). - Ron Chulach, Paul de Bussac, Morris L'hoir, Murray Newton, Guy Wapple (compiler), Rodney Wapple.

EASTEND. Dec. 26; 3 miles on foot in 2 hours and 22 miles by car in 2 hours; temp. $-10^{\circ} \mathrm{C}$, calm; $5 \mathrm{~cm}$ snow; 8 species, $152 \mathrm{in}$ dividuals. (Add: Evening Grosbeak, 25, Dec. 23 and 24). - Lois Clark, Gary Seib (compiler), Barbara, Beryl and Peter Shourounis.

EMMA LAKE. Dec. $20 ; 3-1 / 2$ miles on foot in $3-1 / 2$ hours; temp. $-6^{\circ}$ to $-3^{\circ} \mathrm{C}$, clear, calm; 15 species, 366 individuals. (Add: Goshawk, 1; Downy Woodpecker, 2; Bohemian Waxwing, 10; Red Crossbill, 6). - Kim Godwin.

ENDEAVOUR. Dec. 25; around farm yard and local garbage dump; temp. $-6^{\circ} \mathrm{C}$, calm, overcast; 9 species, 311 individuals. (Add: Downy Woodpecker, 1, Dec. 29; Evening Grosbeak, 5, Jan. 1; Pine Grosbeak, 12, Jan. 1; Common Redpoll, 12, Dec. 27). William Haras.

FORT QU'APPELLE. Dec. $27 ; 35$ miles by car, 2 miles on foot and 3 miles on cross-country skis in $5-1 / 2$ hours; temp. $-1^{\circ}$ to $0^{\circ} \mathrm{C}$, no wind, fine rain falling; $10 \mathrm{~cm}$ of damp snow; 16 species, 551 individuals. (Add: Rock Dove, 6, Dec. 26; American Robin, 1, Jan. 2; Pine Grosbeak, 3, Dec. 25). - Bernard de Vries, Kay de Vries (compiler), Alice Laing, D. Nevard.
GARDINER DAM. Dec. $22 ; 11$ miles on foot in $3-1 / 2$ hours and 45 miles by car in 2 hours; temp. $-10^{\circ}$ to $-6^{\circ} \mathrm{C}$, overcast, wind $0-12$ $\mathrm{mph} ; 8 \mathrm{~cm}$ snow; open water on river for 10 miles below dam and in part of reservoir; 23 species, 333 individuals. (Add: American Wigeon, 1, Jan 3; Horned Lark, 19, Jan. 3 - Wayne Harris, Sheila Lamont). - Bob Godwin, Don Renaud, Wayne Renaud (compiler).

HARRIS. Dec. $20 ; 36$ miles on foot in 23-1/2 hours, 149 miles by car in 20 hours; temp. $-11^{\circ}$ to $-1^{\circ} \mathrm{C}$, clear, wind S-SW, 3-10 mph; $8 \mathrm{~cm}$ snow; 20 species, 2,673 individuals. - Bob Godwin, Bernie and Madeleine Gollop, Wayne Harris, Dale Hjertaas, Mary and Stuart Houston, Sheila Lamont, Don and Wayne Renaud (compiler), Guy and Rodney Wapple, David Surkan.

HEPBURN. Dec. 26; 10 miles by car, 1 mile on foot and about yard, plus sightings at feeder; clear, mild; 9 species, 59 individuals. - Margaret (compiler), Philip, Phyllis and Tena Siemens.

INDIAN HEAD. Jan. $4 ; 17$ miles by car and on foot in 2 hours; temp. $-18^{\circ} \mathrm{C}$, light overcast, calm; $45 \mathrm{~cm}$ snow; 16 species, 1,315 individuals. (Add: Mallard, 30, Jan. 3; Goshawk, 1, Jan. 1; Bald Eagle, 2, Jan 1; Short-eared Owl, 1, Dec. 24; Horned Lark, 1, Dec. 30; Northern Shrike, 1, Jan. 1; Pine Grosbeak, 1, Jan. 1; Common Redpoll, 10, Dec. 30). - Betty and Cec Ashmore, Donald Ayers, Vic Beaulieu family, George Dragan, Marcella Horsman, Jim and Jean Howard, Jim and Bertha Lang, Rose and Roy McLaughlin, Dora Nichols (compiler), Lorne Scott, Mary and Ken Skinner, Fred Skinner, Jean Swinton, Charlie and Ruby Thompson.

KENASTON. Dec. 23; temp. $-12^{\circ}$ to $-7^{\circ} \mathrm{C}$, wind NW 15-20 mph; clear; $5 \mathrm{~cm}$ snow; 7 species, 331 individuals. (Add: Snowy Owl, 1, Dec. 28 and 29; Hairy Woodpecker, 1, Dec. 27; Horned Lark, 1, Jan. 1). - P. Lawrence Beckie (compiler), Joanne, Laurie and Theresa Beckie.

KINDERSLEY. Jan. $3 ; 12$ miles by car; 2 species, 26 individuals. (Add: Bohemian Waxwing, 5, Jan. 1; Common Redpoll, 2, Jan. 1). - Jean Harris.

KUTAWAGAN LAKE (centered 12 miles north of Somans). Jan. 1; 63 miles by car in 4 hours and 1 mile on foot in 1 hour; temp. $-25^{\circ}$ to $-20^{\circ} \mathrm{C}$, cloudy with occasional fog patches, wind SW $9 \mathrm{mph} ; 18 \mathrm{~cm}$ snow; 7 species, 996 individuals. - Wayne Harris, Sheila Lamont.

LAST MOUNTAIN LAKE (management unit and Immediate area). Dec. 28; 95 miles by car in 6 hours and 1 mile on foot in 1 hour; temp. 
Species recorded from more than one locality on Count day

\begin{tabular}{|c|c|c|c|c|c|c|c|c|c|c|c|c|c|}
\hline \multirow{2}{*}{\multicolumn{14}{|c|}{ Canada Gorese }} \\
\hline & & & & & & & & & & & & & \\
\hline Mallard & & & & & & 6 & & 178 & & & & & \\
\hline \multicolumn{14}{|l|}{ Pintail } \\
\hline \multicolumn{14}{|l|}{ Lesser Scaup } \\
\hline Common Goldeneye & & & & & & & & 28 & & & & & \\
\hline \multicolumn{14}{|l|}{ Common Merganser } \\
\hline Goshawk & & & & & & 1 & & & & & & & \\
\hline Golden Eigle & & & & & & 2 & & & & & & & \\
\hline \multicolumn{14}{|l|}{ Biald Eagle } \\
\hline Merlin & 1 & & & & 1 & & & & & & & & \\
\hline Ruffed Grouse & 2 & & & 5 & & & & 5 & & & & & 2 \\
\hline Sharp-lailcd Grouse & & & 27 & 22 & 20 & 87 & & & 4 & & 3 & & \\
\hline Ring-neek. Pheasant & & & & & & & & & & & & 2 & \\
\hline Gray Partridge & & & 2 & & & & & & & 15 & 42 & 4 & \\
\hline \multicolumn{14}{|l|}{ Ameriean Cont } \\
\hline Rock Dove & & & 48 & & & 70 & & & 47 & 8 & 67 & & \\
\hline \multicolumn{14}{|l|}{$\overline{\text { Mourning Dove }}$} \\
\hline Great Horned Owl & 1 & & 2 & 1 & & 1 & & & 1 & & 2 & & \\
\hline Snowy Owl & & & & & 1 & & & & 2 & & 2 & & \\
\hline \multicolumn{14}{|l|}{ Short-eared Ow! } \\
\hline \multicolumn{14}{|l|}{ Common Flieker } \\
\hline Pilcated Wordpecker & & & & & & & & 1 & & & & & 1 \\
\hline Hairy Woodpeeker & & 2 & 2 & 2 & & 1 & 1 & & 1 & 1 & & 2 & 2 \\
\hline Downy Woodpecker & & 1 & & 4 & & 3 & & & & 2 & 1 & & \\
\hline N. 3-1. Woodpecker & & & & 1 & & & & & & & & & 1 \\
\hline Horned Lark & & & & & & & & & $T$ & 2 & & & \\
\hline Gray Jay & 1 & & & & & & & & & & & & 12 \\
\hline Blue Jay & & & & 3 & & & & 2 & & & & & 9 \\
\hline Black-billed Magpic & 2 & 1 & 139 & 24 & 23 & 16 & 2 & 8 & 42 & 17 & 89 & 23 & 3 \\
\hline Common Raven & & & & 2 & & & 1 & 4 & & & & & 7 \\
\hline Bl.-cap. Chickadee & 2 & 4 & 33 & 14 & 2 & 1.5 & 2 & 7 & 27 & & 8 & 10 & 46 \\
\hline Boreal Chickadec & 1 & & & 1 & & & & & & & & & 10 \\
\hline W-breasted Nuthatch & & & & & & & & & & & & & 2 \\
\hline R-breasted Nuthatch & & & & 1 & & & & & & & & & 7 \\
\hline Brown Creper & & & & & & & & & & & & & 4 \\
\hline \multicolumn{14}{|l|}{ American Robin } \\
\hline \multicolumn{14}{|l|}{ Townsend's Sellitaire } \\
\hline Gold.-cr. Kinglet & & & & 4 & & & & & & & & & \\
\hline Bohemian Waxwing & 18 & & 111 & 12 & & 4 & & & 22 & 1.5 & 55 & & \\
\hline \multicolumn{14}{|l|}{ Cedar Waxwing } \\
\hline Northern Shrike & & & & & & 1 & & & & & & & \\
\hline Starling & & & & & & & & & & & 4 & & \\
\hline House Sparrow & & 6 & 871 & 51 & 150 & 127 & 15 & 3 & 209 & 167 & 1381 & 11 & \\
\hline Rusty Blackbird & & & & & & & & & & & 1 & & \\
\hline Evening Grosbeak & & & 6 & 1 & & 10 & 5 & & & & 4 & & \\
\hline Pine Grosheak & & & 4 & 61 & & 3 & & 4 & 2 & & & & \\
\hline Hoary Redpoll & & & 1 & 2 & & & & & & & 5 & & \\
\hline Common Redpoll & & 14 & 307 & 455 & 402 & 181 & & 3 & 76 & 36 & 410 & 75 & 30 \\
\hline \multicolumn{14}{|l|}{ Pine Siskin } \\
\hline Red Crosslill & & & & 17 & & 1 & & & & & & & \\
\hline W-winged Crosshill & & & 1 & 32 & & & & & & & & & 230 \\
\hline \multicolumn{14}{|l|}{ Dark-cyed Junco } \\
\hline Snow Bunting & 25 & 30 & & 25 & 1 & & & & 758 & & 1.5 & 25 & \\
\hline Single Species & & & & & & & & & & & & & \\
\hline Total Species & 9 & 7 & 14 & 22 & 8 & 17 & 6 & 11 & 13 & 9 & 16 & 8 & 1.5 \\
\hline
\end{tabular}


Species recorded from more than one locality on Count day

Canada Goose

Mallard

Pintail

Lesser Sciup

Common Goldeneye

Common Merganser

Goshawk

Golden Eagle

Bald Eagle

Merlin

Ruffed Grouse

Sharp-tailed Grouse

Ring-neck. Pheasint

Graly Partridge

American Coot

Rock Dove

Mourning Dove

Great Horned Owl

Snowy Owl

Short-cared Owl

Comınon Flicker

Pileated Woodpceker

Hairy Woodpecker

Downy Woodpecker

N. 3-1. Wordpecker

Horned Lark

Gray Jay

Blue Jaly

Black-billed Magpic

Common Raven

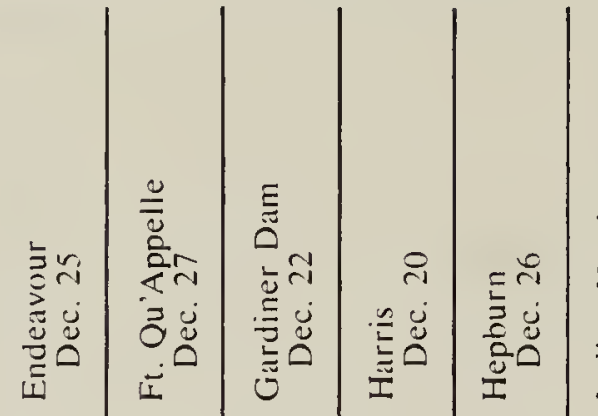

焉

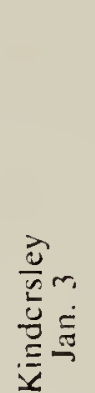

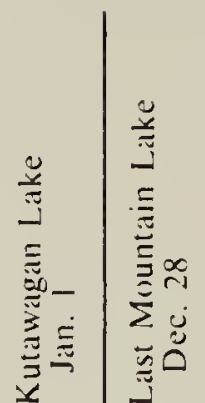

\begin{tabular}{r|r|r|r|r|r|}
\hline & 1 & & & & \\
\hline & 30 & 16 & & & \\
\hline & + & 6 & & & \\
\hline & & 3 & & & \\
\hline & & & 2 & &
\end{tabular}

\begin{tabular}{r|r|r|r|r|r|}
\hline & 1 & & & & \\
\hline & 30 & 16 & & & \\
\hline & + & 6 & & & \\
\hline & & 3 & & & \\
\hline & & & 2 & & \\
\hline
\end{tabular}

-

Bl.-cap. Chickaldec

Boreal Chickadee

W-breasted Nuthatch

R-brcalsted Nuthatch

Brown Creeper

American Robin

Townsend's Solitaire

Gold.-cr. Kinglet

Bohemian Waxwing

Cedar Waxwing

Northern Shrike

Starling

House Sparrow

Rusty Blackbird

Evening Grosbeak

Pine Grosheak

Hoary Redpoll

Common Redpoll

Pine Siskin

Red Crosshill

W-winged Crossbill

Dark-cyed Junco

Snow Bunting

Single Species

Total Species

\begin{tabular}{ll|l|l|}
\hline & & & \\
\hline & & &
\end{tabular}

\begin{tabular}{|c|c|c|c|c|}
\hline & & & & \\
\hline & & & 1 & \\
\hline & & & 1 & \\
\hline 1 & & +1 & 217 & \\
\hline & & & & \\
\hline & & & 48 & \\
\hline & & 1 & & \\
\hline 1 & & 12 & 78 & \\
\hline & & & & \\
\hline 1 & & 3 & 6 & 1 \\
\hline
\end{tabular}

\begin{tabular}{ll|l|l|}
\hline & & & \\
\hline & & & \\
\hline
\end{tabular}

\begin{tabular}{|r|r|r|r|r|r|r|r|r|r|}
\hline & & & & & & & \\
\hline 2 & 2 & 1 & 3 & 1 & 4 & & \\
\hline & 1 & 1 & 1 & & 5 & & \\
\hline 6 & & & & & & & \\
\hline 29 & 1 & 15 & 247 & 2 & 14 & 14 & \\
8 & 7 & 6 & 39 & 5 & 13 & &
\end{tabular}
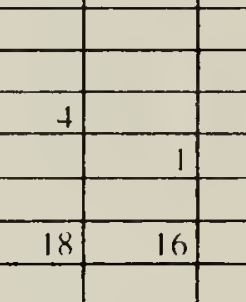

$+$

$+$

\begin{tabular}{l|l|l}
12 & +
\end{tabular}

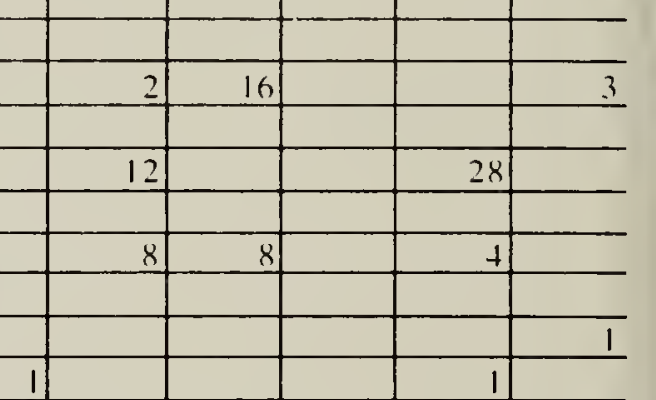

$+$

\begin{tabular}{|c|c|c|c|c|c|c|c|c|c|c|c|c|}
\hline & & & 1 & & & & & & & & & \\
\hline & 219 & 32 & 178 & 10 & 25 & & & & & & 34 & \\
\hline & 21 & & & & & & & & & & & \\
\hline & & & & & & & & & & & & \\
\hline & & 6 & 1 & & & & & & 1 & & & \\
\hline 81 & 180 & 14 & $9+3$ & 12 & 1000 & 7.5 & & 155 & $4+7$ & 2.5 & 122 & 40 \\
\hline & & & & & & & & & & & & \\
\hline & 20 & 3.5 & 14 & & 7 & & & & & 10 & 2 & \\
\hline & & 12 & & 12 & & & & & & & & 9 \\
\hline & 10 & & 2 & & & & & & 2 & & & \\
\hline & 48 & 86 & 76.5 & & & 71 & & 692 & 588 & & 105 & 275 \\
\hline & & & & & & & & & & & & \\
\hline & & & & 1 & & & & & & & & \\
\hline & & & & & 4 & & & & & & & \\
\hline & & & & & 1 & & & & & & & \\
\hline 182 & & 70 & 122 & 15 & 200 & 1.50 & 25 & 81 & 226 & & 1186 & 121 \\
\hline & 1 & 1 & & & & & & & & & & \\
\hline 9 & 16 & 2.3 & 20 & y & 16 & 7 & 2 & 7 & 10 & 3 & 11 & 11 \\
\hline
\end{tabular}




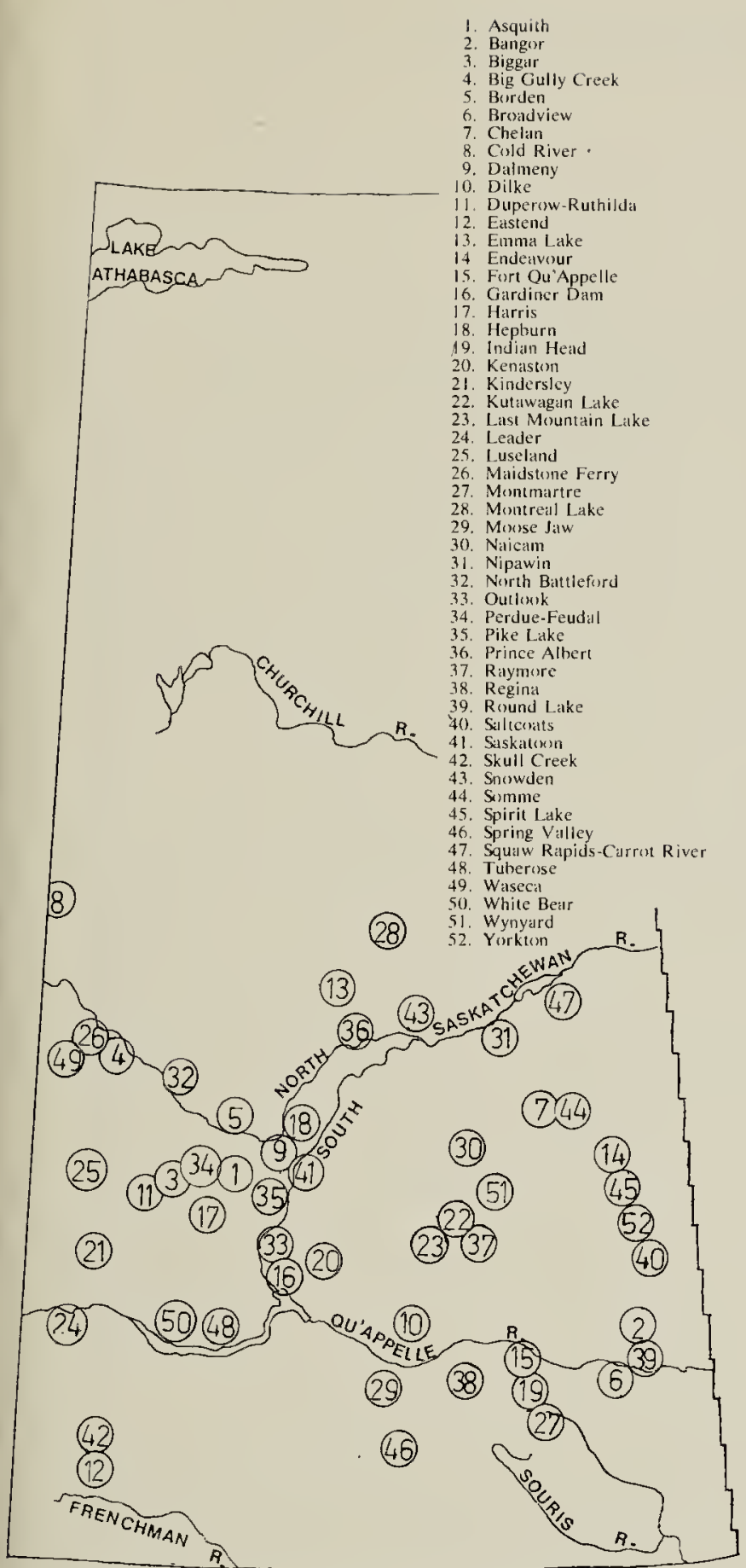

Map prepared by Wayne Harris $-15^{\circ}$ to $-5^{\circ} \mathrm{C}$, clear, wind SW 13-22 mph; 20 $\mathrm{cm}$ of snow; 10 species, 1,328 individuals. - Wayne Harris, Sheila Lamont.

LEADER. Jan. 4; around the farm for 3 hours; temp. $-12^{\circ} \mathrm{C}$, clear; $30 \mathrm{~cm}$ snow; 3 species, 47 individuals. (Add: Sharp-tailed Grouse, 20, Dec. 24; Ring-necked Pheasant, 1, Dec. 21; Dark-eyed Junco, 1, Dec. 24). - Daisy D. Meyers.

LUSELAND. Dec. 24; 73 miles by car and 2 miles on foot in 5 hours; temp. $-1{ }^{\circ} \mathrm{C}$, clear: $10 \mathrm{~cm}$ snow; 11 species, 1,527 individuals. (Add: Golden Eagle, 1, Dec. 27). - K. B. Finley, K. J. Finley, B. Holton.

MAIDSTONE FERRY (21 miles $\mathbf{N}$ of Maidstone). Dec. 23; 30 miles by car in 3 hours and 2 miles on foot in 1 hour; temp. $-20^{\circ}$ to $-6^{\circ} \mathrm{C}$, cloudy, wind SW $10 \mathrm{mph} ; 25 \mathrm{~cm}$ snow; 11 species, 475 individuals. (Add: Evening Grosbeak, 1, Dec. 24). — Wayne Harris, Sheila Lamont.
MONTMARTRE. Dec. $26 ; 2$ miles on foot in 3 hours; temp. $-10^{\circ} \mathrm{C}$, sunny with cloudy periods; $28 \mathrm{~cm}$ snow; 11 species, 415 individuals. - Edwin Lesiuk.

MONTREAL LAKE. Jan. 4; 35 miles by car in 4 hours and 3 miles on foot in 2 hours; temp. $-25^{\circ}$ to $-20^{\circ} \mathrm{C}$, cloudy, wind NW 15 to 35 mph; $30 \mathrm{~cm}$ snow; 12 species, 128 individuals. - Wayne Harris, Sheila Lamont.

MOOSE JAW. Dec. 26; 96 miles by car and 8 miles on foot; temp. $0^{\circ}$ to $3^{\circ} \mathrm{C}$; light snow cover; 21 species, 2,300 individuals. (Add: White-winged Crossbill, 6, Dec. 23). Bob Beattie, Russ Dennison, Alice and Carl Ellis, Doug Francis, Ruth Hilling, John Horton, Pat Kern, Cy and Leith Knight (compiler), Moray Lewis, Molly Ritchie, Jean Thomson.

NaICAM. Dec. 28; 1 mile on foot; temp. $-10^{\circ} \mathrm{C}$, wind $20 \mathrm{mph} ; 3$ species, 37 individuals. - Ron Jensen.

NIPAWIN. Jan. 3 ; temp. $-33^{\circ} \mathrm{C}$, bitter $\mathrm{N}$ wind; 11 species, 611 individuals. - Mrs. Harvey Christiansen, John Comer, Stan Riome (compiler).

NORTH BATTLEFORD. Dec. $28 ; 6$ miles on foot in $2-1 / 2$ hours; temp. $-3^{\circ} \mathrm{C}$, partly cloudy, calm; 6 species, 55 individuals. - Ralph T. Cowell.

outLook. Dec. 23; 4 species, 536 individuals. (Add: Black-billed Magpie, 2, Dec. 30; Black-capped Chickadee, 2, Dec. 30; Bohemian Waxwing, 40, Dec. 30; Common Redpoll, 2, Dec. 30). - Harold Kvinge.

PERDUE-FEUDAL. Jan. 4; 76 miles by car in 3 hours, 7 miles on foot in 4 hours; temp. $-13^{\circ}$ to $-8^{\circ} \mathrm{C}$, wind SW 0 to $10 \mathrm{mph}$, overcast in AM light fog and snow in PM; $28 \mathrm{~cm}$ snow; 10 species, 1,724 individuals. - Ron Chulach, Morris L'hoir, Guy Wapple (compiler), Rodney Wapple.

PIKE LAKE. Dec. 24; 28 miles by car in 3 hours, $1-1 / 2$ miles on foot in $1 / 2$ hour; temp. $-7^{\circ} \mathrm{C}$, wind SE $5 \mathrm{mph}$, partly cloudy; $5 \mathrm{~cm}$ snow; 11 species, 329 individuals. Robbie and Ron Bobowski, Mr. and Mrs. M. E. Christensen, John and Stan Shadick.

PRINCE ALbert. Dec. 26; within 7 miles of the city, and at 12 feeding stations; 16 species, 615 individuals. - Ansgar and Christie Aschim, David and Peter Surkan.

RAYMORE. Dec. 29; 107 miles by car in 7 hours, 2 miles on foot in 2 hours; temp. $-15^{\circ}$ to $-3^{\circ} \mathrm{C}$, cloudy with occasional clear patches, wind NW $0-15 \mathrm{mph} ; 25 \mathrm{~cm}$ snow; 19 species, 1,367 individuals. - Chas, Greta and Wayne Harris, Sheila Lamont.

REGiNA. Dec. 26; 522 miles by car in 38 hours, 50 miles on foot in 32 hours; temp. 
Species recorded from more than one locality on Count day

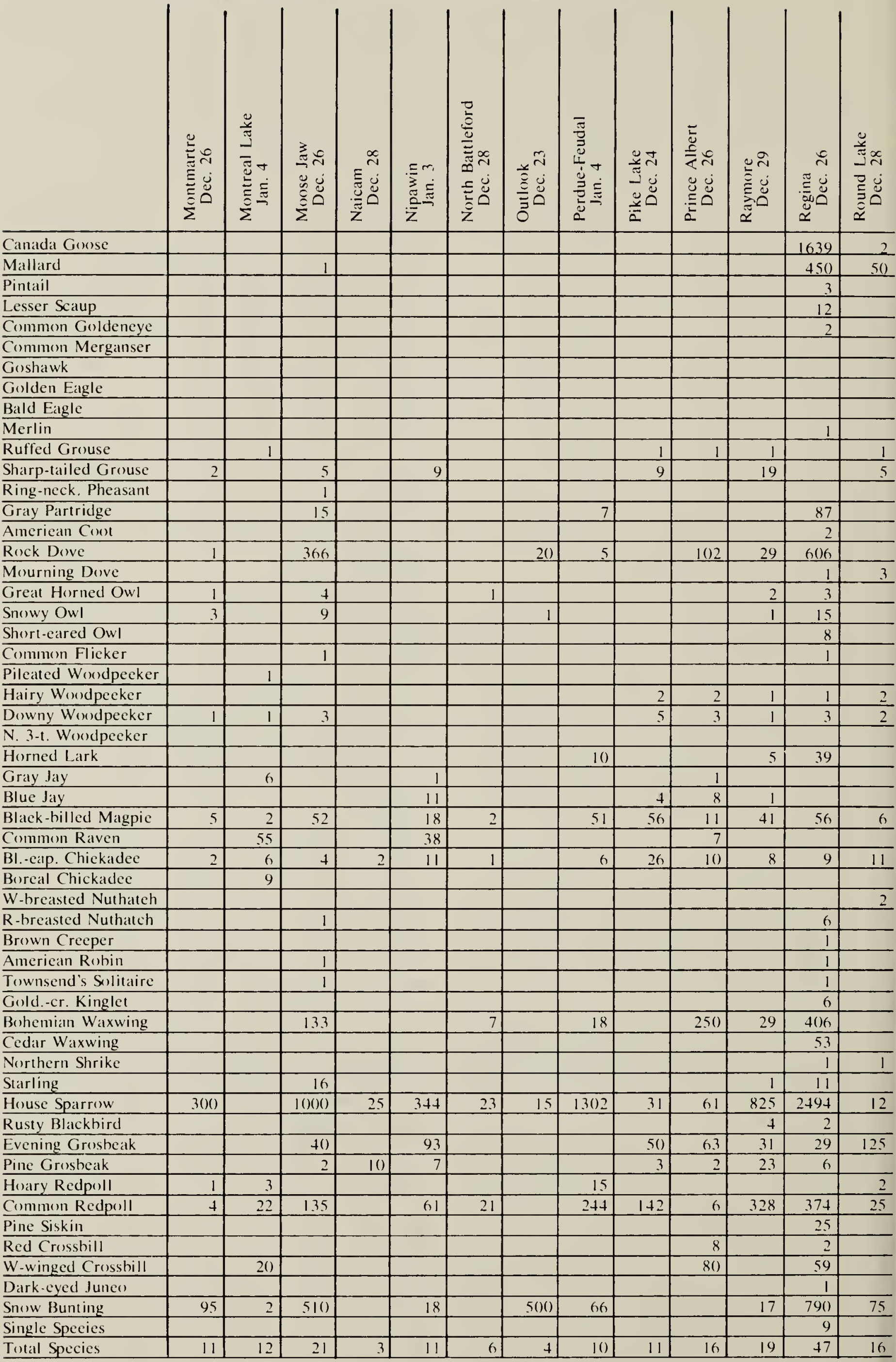


Species recorded from more than one locality on Count day

anada Goose
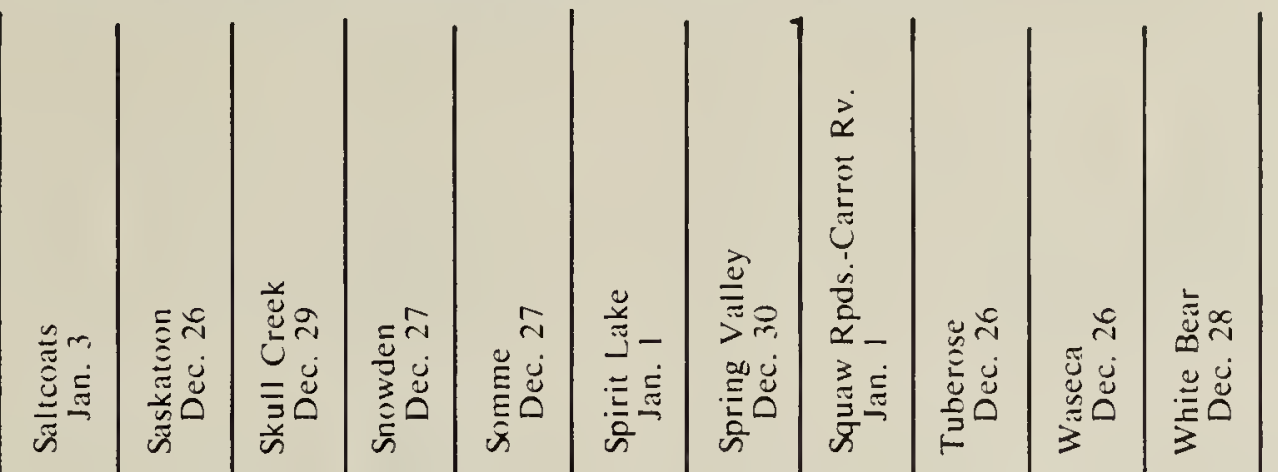

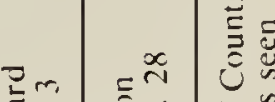

lallard

38

intail

esser Scaup

ommon Goldeneye

ommon Merganser

joshawk

iolden Eagle

ald Eagle

Terlin

uffed Grouse

harp-tailed Grouse

ing-neek. Pheasant

iray Partridge

meriean Coot

ock Dove

fourning Dove

reat Horned Owl

nowy Owl

hort-eared Owl

ommon Flicker

ileated Woodpeeker

Aiiry Woodpeeker

ouwny Wordpeeker

1. 3-1. Wordpeeker

orned Lark

ray Jay

lue Jay

lack-billed Magpie

ommon Raven

l.-eap. Chiekadee

oreal Chickadee

-breasted Nuthateh

- breasted Nutlatteh

rown Creeper

meriean Robin

ownsend's Solitaire

old.-er. Kinglet

ohemian Waxwing

edar Waxwing

orthern Shrike

arling

ouse Sparrow

usty Blackbird

vening Grosbeak

ine Grosbeak

Oary Redpoll

(mm11!11 Redpoll

inc Siskin

ed Crossbill

-winged Crossbill

\begin{tabular}{r|r}
1 & -2 \\
\hline 3 & \\
203 &
\end{tabular}

\begin{tabular}{|r|r|r}
\hline & 38 & \\
\hline & 1 & \\
\hline & 3 & \\
\hline & 203 & \\
\hline & & \\
\hline
\end{tabular}

$\mid$

\begin{tabular}{|l|l|l|}
\hline & & \\
\hline & 5 &
\end{tabular}

\begin{tabular}{|r|r}
\hline 2 & 10 \\
\hline 16 & 44 \\
\hline & 8 \\
\hline
\end{tabular}

ark-eyed Junco

low Bunting

ngle Speeies

otal Speeies

\begin{tabular}{r|r|r|}
\hline 10 & \\
44 & 8 &
\end{tabular}

\begin{tabular}{r|r}
40 \\
8 \\
\hline 40
\end{tabular}

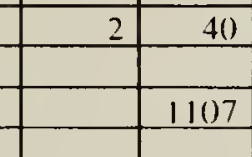

\begin{tabular}{|l|r|r}
\hline & 3 & \\
\hline & 3 &
\end{tabular}

.

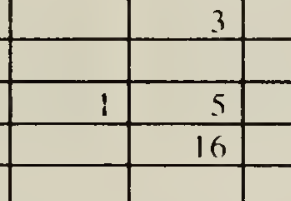

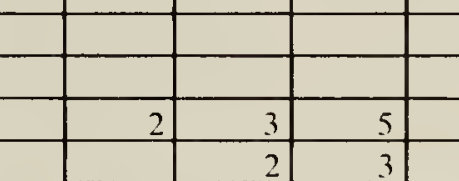

\begin{tabular}{l|l}
\hline & \\
\hline &
\end{tabular}

\begin{tabular}{l|l|}
\hline 12 & \\
\hline & \\
\hline
\end{tabular}

हे

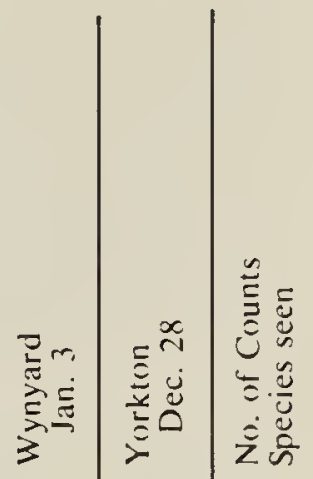

\begin{tabular}{r|r|}
\hline 42 & \\
\hline 2 & \\
\hline &
\end{tabular}

\begin{tabular}{|l|l|l|l|l|}
\hline & & & & 3 \\
\hline & & & & 2 \\
\hline & & & & 2 \\
\hline
\end{tabular}


Species recorded from only 1 locality on Count day

$\begin{aligned} \text { No. } & \text { Species } \\ 1 & \text { Eared Grebe } \\ 2 & \text { Mute Swan } \\ 1 & \text { Blue-winged Teal } \\ 1 & \text { American Wigeon } \\ 2 & \text { Northern Shoveler } \\ & \\ 2 & \text { Redhead } \\ 2 & \text { Canvasback } \\ 1 & \text { Ruddy Duck } \\ 1 & \text { Prairie Falcon } \\ 1 & \text { Peregrine Falcon } \\ 3 & \text { Glaucous Gull } \\ 1 & \text { Black-backed Three-toed Woodpecker } \\ 1 & \text { Brewer's Blackbird } \\ 2 & \text { Tree Sparrow } \\ 21 & \text { Lapland Longspur }\end{aligned}$

$-11^{\circ}$ to $-7^{\circ} \mathrm{C}$, partly cloudy, clearing in PM, wind ESE 6 to $12 \mathrm{mph} ; 47$ species, 7,239 individuals. - Jessie Bailey, Margaret Belcher, Mr. and Mrs. Tom Beveridge, Eric Cooke, Mae Cross, Robyn Donison, Dick and Maureen DuWors, David Duffis, Lucy Eley, Mr. and Mrs. Murray Fines, Wayne Gemmell (compiler), Al Harrison, Val Harrison, Jim Hines (compiler), Rose Hines, Jim and Shirley Jowsey, Darlene Kauk, Dave Kelley, Eric Lang, Tony Lang, George Ledingham, Madelaine McGinnis, Will Marchuk, Helen Morrison, Sally Moss, Keith Neufeld, Roxanne Pelletier, Connie Pratt, Joe Roberts, Con Rosenfeld, Anne Russon, Bill Russon, John Russon, Rick St. Pierre, Allen and Diane Smith, Joyce Swenson, Frank Switzer, Jay Van Oostdam. Christophe Wilhelm, Pierre Wilhelm, Rita Wilhelm, Cheryl Zagozeski.

ROUND LAKE. Dec. 28; 10 miles along lake and at feeder; mild; 16 species, 324 individuals. - Doug Francis.

Saltcoats. Jan. $3 ; 44$ miles by car in $3-1 / 4$ hours; temp. $-23^{\circ} \mathrm{C}$, calm, sunny; 10

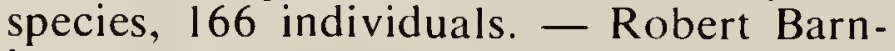
hart.

SASKATOON. Dec. $26 ; 296$ miles by car in 41 $1 / 2$ hours, 75 miles on foot in 48 hours; temp. $-20^{\circ}$ to $-10^{\circ} \mathrm{C}$, wind light, mostly cloudy; 34 species, 19,371 individuals. (Add: Brown Creeper, 1, Dec. 24). - Ann Marie and Norman Ash, Ron Bobowski, Murray and Ron Bremner, Pern Cordery, Cliff Finlay, Doug Finlay, Muriel Galloway, Marie Gillespie, Bernie, Madeleine and Michael Gollop, Jack and Louise Greaves, Wayne Harris, Jim Heffernan, Clarence, Dave, Don, Mary, Stan, and Stuart Houston (compiler), Grev Jones,
Locality

Map No.

Regina

Regina 38

Regina $\quad 38$

Regina 38

Regina 38

Regina $\quad 38$

Regina $\quad 38$

Regina 38

White Bear 50

Squaw Rapids-Carrot River $\quad 47$

Gardiner Dam $\quad 16$

Somme 44

Fort Qu'Appelle 15

Skull Creek 42

Regina

Sheila Lamont, Keith Martens, Don, Elizabeth, Joanne and Margaret McRobbie, Betty Mundy, Arnold Nijssen, Pat O'Neil, Stuart Rasmussen, Wayne Renaud, Adam Schmidt, John, Mary and Stan Shadick, Alan, Edward and Gary Smith, Sheldon Snatinsky.

SKULL CREeK. Dec. 29; on foot 1-1/2 miles up the creek; temp. $-12^{\circ} \mathrm{C}$, light wind, clear; 10 species, 164 individuals. - Helen and Ray Schuler.

SNOWDEN. Dec. 27; 7 miles by car and 4-1/2 miles on foot; temp. $-1{ }^{\circ} \mathrm{C}$, freezing rain, wind SW $5 \mathrm{mph} ; 9$ species, 86 individuals. - D. A. Jacura, Juliana Soroka (compiler).

SOMME. Dec. 27; 20 miles by car and 2 miles on foot; temp. $-5^{\circ} \mathrm{C}$; 20 species, 145 individuals. (Add: Rock Dove, 10, Dec. 26; Horned Lark, 1, Dec. 31: Brown Creeper, 1, Dec. 29; White-winged Crossbill, 2, Dec. 29). - David Black, Donald and Ronald Hooper.

SPIRIT LAKE. Jan. 1: 53 miles by car in 4 hours, 2 miles on foot in $1-1 / 2$ hours; temp. $-20^{\circ}$ to $-14^{\circ} \mathrm{C}$, wind light, cloudy, clearing in $\mathrm{PM} ; 15 \mathrm{~cm}$ snow; 14 species, $48 \mathrm{l}$ individuals. (Add: Goshawk, 1, Dec. 22; Great Horned Owl, 3, Dec. 29; Common Raven, 5, Dec. 21; Northern Shrike, I, Jan. 3: Snow Bunting, 2, Jan. 4). - Bill and Joyce Anaka, Mrs. Gunn.

SPRING VALLEY. Dec. $30 ; 34$ miles by car and around farm; temp. $-5^{\circ}$ to $-1{ }^{\circ} \mathrm{C}$, wind light, overcast; about $10 \mathrm{~cm}$ snow; 11 species, 1,101 individuals. (Add: Golden Eagle, 1, Dec. 23: Prairie Falcon, 1, Dec. 26; Bohemian Waxwing, 18, Dec. 20; Graycrowned Rosy Finch, 1, Dec. 30). Adeline, Allan, Flossie (compiler), Larry and Nick Bogdan. 
SQUAW RAPIDS-CARROT RIVER. Jan. 1; 8 hours: temp. $-21{ }^{\circ} \mathrm{C}$, calm, overcast to $4 \mathrm{PM} ; 18$ species, 1,749 individuals. - John Comer, Terry Klassen, Roland Lockhart, Gladys and Stan Riome (compiler).

TUBEROSE. Dec. 26; 40 miles by car in 2 hours; temp $-6^{\circ}$ to $-4^{\circ} \mathrm{C}$, calm, sunny; 5 species, 115 individuals. (Add: Golden Eagle, 1, Dec. 20). - Cliff Matthews.

WASECA. Dec. $26 ; 2-1 / 2$ miles on foot around farmstead and marsh area; temp. $-4^{\circ} \mathrm{C}$, sunny, light $\mathrm{SE}$ wind; $20 \mathrm{~cm}$ snow; 10 species, 156 individuals. (Add: Common Redpoll, 50, Dec. 22 and 24; Snow Bunting, 100, Dec. 22 and 24). - Darcy, Colleen, Maureen, Michelle, Russell and Stuart Bexson, Christine Pike (compiler).

\section{WANTED: NORTHERN MANITOBA NESTLINES}

An interesting report comes from Klon Peterson, a young fellow who lives with his family at Prospector Ranger Tower, 11 miles north of The Pas, Manitoba. Klon set out some bluebird nest boxes in 1975 and, although he had no bluebird nestings, the birds were seen in that area, which seems to be quite far north. Four Tree Swallows nested in the boxes, hatching 16 young. Wrens also used one box. We hope Klon will find time to build more nest boxes this winter and report to us again next year.

If anyone living in that area or south of The Pas is interested in establishing a nestline, it would be a worthwhile project because we would like to find out how far north our bluebirds do go. One next box at Wabowden, some 160 miles northeast of The Pas, was set out but owing to flooding it was not checked. Do we have any interested readers in that area? - Jim Spear. Russell, Manitoba.
WHITE BEAR. Dec. 28: 2 miles by car and 4 $1 / 2$ miles on foot; temp. $-1{ }^{\circ} \mathrm{C}$, cloudy, light west wind; 5 to $15 \mathrm{~cm}$ crisp snow: 9 species, 363 individuals. (Add: Golden Eagle, 2, Dec. 29). - Oran Cates, Anne, Chris, Eric and Mark Christenson, Leroy Clark, Gary, Gerald, Laine and Sig Jordheim (compiler), David Lowe, Kenny Markella, Dan Schuler, Doug Stepples.

WYNYARD. Jan. 3; 5 hours and 10 miles by car; temp. $-29^{\circ} \mathrm{C}$, sunny, calm; 8 species, 682 individuals. - John and Sherry Gulley.

YORKTON. Dec. 28 ; temp. $-2^{\circ} \mathrm{C}$, wind 10 mph, clear; 9 species, 405 individuals. Margaret Bromley,(compiler), P. Pawluck, T. Pawluck.

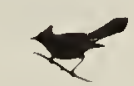

\section{FORT SMITH, N.W.T., CHRISTMAS BIRD COUNT}

\author{
Compiled by ERNIE KUYT, \\ Box 508, Fort Smith, N.W.T.
}

DATE: Dec. 28, 1975.

WEATHER: Cloudy with sunny intervals; temperature about $0^{\circ} \mathrm{C}$; calm; snow depth about $40 \mathrm{~cm}$; daylight 9 AM to 4 PM.

ROUTES COVERED: Fort Smith to Fox Holes road intersection; Fort Smith along Pine Lake road to Salt River; Fort Smith along Hay Camp road to Wood Buffalo National Park border. 100 miles by car in 3 hours.

BIRDS SEEN: Spruce Grouse, 13: Ruffed Grouse, 1; Rock Dove, 9; Hawk Owl, 1; Great Gray Owl, 1: Gray Jay, 31: Common Raven, 178: Boreal Chickadee, 1: House Sparrow, 10; Snow Bunting, 7. 10 species, 252 birds. Add: Willow Ptarmigan, 1, Dec. 27: Pileated Woodpecker, 1, Dec. 21: Downy Woodpecker, 1, Dec. 27; Black-capped Chickadee, 2, Dec. 27.

CONTRIBUTORS: Don, Isabel and Tom Brannigan, Dan and Linda Graham, Brian, Jeremy, Joyce and Tyler Johnson, Elsie, Ernie (compiler), Jonathan and Pamela Kuyt, Bernadette McGill, Randy Mitchell. 